\title{
Breast Magnetic Resonance Imaging: Initial Experience in Kuwait
}

\author{
Hanaa Al-Khawari ${ }^{\mathrm{a}}$ Agnes Kovacs $^{\mathrm{b}}$ Reji Athyal ${ }^{\mathrm{b}}$ Huda Al-Manfouhi ${ }^{\mathrm{c}}$ \\ Mohammed Salah Fayaz ${ }^{\mathrm{e}}$ John Patrick Madda ${ }^{\mathrm{d}}$ \\ ${ }^{a}$ Department of Clinical Radiology, Faculty of Medicine, Kuwait University, Departments of b Clinical Radiology, \\ 'Surgey and d Pathology, Al-Amiri Hospital Kuwait, and 'Department of Medical Oncology, Kuwait Cancer Control \\ Center, Kuwait
}

\author{
Key Words \\ Breast cancer $\cdot$ Breast neoplasm $\cdot$ Magnetic resonance \\ imaging $\cdot$ Xeromammography
}

\begin{abstract}
Objective: To report our initial experience of breast magnetic resonance imaging (MRI) in Kuwait in order to identify and characterize breast lesions. Subjects and Methods: In 58 patients ranging in age from 25 to 64 years, breast MRI was performed as a problem-solving tool (29); for suspicious local relapse of the treated breast (6); to search for a primary breast cancer in patients with metastatic axillary lymph nodes (5); for local staging of breast cancer (5); breast implants (6); screening in high-risk patients (3), and differentiation between inflammation and inflammatory carcinoma (4). Sagittal fat-saturated $T_{2}$ and axial $T_{1}$ images were obtained before, and axial fat-saturated $\mathrm{T}_{1}$ and dynamic sagittal fat-saturated $\mathrm{T}_{1}$-weighted images after contrast enhancement in a 1.5-tesla closed magnet. The diagnostic criteria were based on the morphology and kinetics of the lesion. Findings were validated by tissue sampling or radiological follow-up. Results: Seventy breast lesions (25 malignant, 38 benign and 7 lesions detected by MRI only) were identified in the 58 patients. The sensitivity, specificity, and positive and negative predictive values of MRI in diagnosing malignant breast lesions were 96, 67, 71 and 95\%, respectively,
\end{abstract}

while the accuracy was $80 \%$. Conclusion: This initial experience is comparable to other published data. Future plans for improving image spatial resolution and MR-guided procedures have been taken into consideration.

Copyright $\odot 2009$ S. Karger AG, Basel

\section{Introduction}

Breast cancer is the most common malignant tumor worldwide among women except in Japan [1]. The incidence of breast cancer among Kuwaiti and non-Kuwaiti women is 33 and 35 per 100,000, respectively, according to the WHO cancer incidence statistics from the International Agency for Research on Cancer (IARC) and the International Association for Cancer Registries [1]. X-ray mammography and high-frequency breast ultrasonography (US) are the primary diagnostic tools for the evaluation of breast cancer, but accuracy is still limited in some situations [2]. During the past few years, breast magnetic resonance imaging (MRI) has become an integral component in diagnosing and staging breast malignancies [3-5]. The aim of this study was to report our initial experience of breast MRI in Kuwait in identifying and characterizing breast lesions of patients with suspicious mammographic and/or ultrasonic lesions and to correlate the findings with tissue sampling. For those patients for

\section{KARGER}

Fax +4161306 1234

E-Mail karger@karger.ch

www.karger.com
(C) 2009 S. Karger AG, Basel

1011-7571/09/0182-0143\$26.00/0

Accessible online at:

www.karger.com/mpp
Dr. Hanaa Al-Khawari

Department of Clinical Radiology, Faculty of Medicine, Kuwait University PO Box 24923

13110 Safat (Kuwait)

Tel. +965 498 6411, Fax +965 533 0473, E-Mail hkhawari@gmail.com 
whom a tissue sample was not obtained, follow-up with mammography, US or breast MRI was done for at least 1 year.

\section{Subjects and Methods}

The study was conducted in the Al-Amiri Hospital during the period from May 2005 to September 2006. Standard two-view digital mammography (Selenia; Lorad, Danbury, Conn., USA), with additional views if required, were obtained together with high-resolution breast US (EnVisor; Philips, Seattle, Wash., USA) for patients with clinically, mammographically or ultrasonographically suspicious breast lesions. Breast MRI was performed with a 1.5-tesla closed magnet (Excite; GE, Milwaukee, Wisc., USA) using a dedicated 4-channel phased-array breast coil for 58 consecutive patients ranging in age from 25 to 64 years. The patients were referred to our MRI unit to assess mammographically equivocal breast lesions (29) and breast implants (6); for suspicion of local relapse in a treated breast (6); to search for primary breast cancer in patients with metastatic axillary lymph nodes (5); for local staging of breast cancer (5; suspicion of multiple lesions at standard imaging, dense breasts); differentiation between inflammatory benign lesions and inflammatory carcinoma (4), and MRI screening in high-risk patients (3).

\section{Preparation and Positioning of the Patients}

Breast MRI was performed during days 6-16 of the menstrual cycle or after stopping hormone replacement therapy for 4-6 weeks to avoid false-positive enhancing lesions during the peak hormonal level of the cycle. To detect recurrent malignant lesions, breast MRI was performed 6-8 weeks after surgery and at least 9 months (preferably 12 months) after radiotherapy. A 20 -gauge intravenous line was inserted at the dorsum of the hand before positioning the patient on the table to ensure lack of movement between scans. The patient lay in a prone position on a platform placed in the MR imager that allows the breasts to be in a dependent position.

\section{Protocol}

High-resolution axial $\mathrm{T}_{1}$-weighted $3 \mathrm{D}$ SPGR volume images (number of excitations, NEX, 1; voxel size $1.0 \mathrm{~mm}^{3}$, and spatial resolution of $0.7 \times 7 \times 2.5 \mathrm{~mm}$ slice thickness) and axial fatsaturated $\mathrm{T}_{2}$-weighted 2D FSE images (NEX 4; voxel size $8 \mathrm{~mm}^{3}$, and spatial resolution of $1.3 \times 1.3 \times 5 \mathrm{~mm}$ slice thickness) were obtained prior to contrast injection, and axial or coronal fat-saturated $\mathrm{T}_{1}$-weighted high-resolution $3 \mathrm{D}$ SPGR dynamic images (NEX 1; voxel size $7 \mathrm{~mm}^{3}$, and spatial resolution of $1.4 \times 1.4 \times$ $4 \mathrm{~mm}$ slice thickness) were then performed with an acquisition time that did not exceed $60 \mathrm{~s}$ per scan. The scan was repeated 6 times after contrast medium injection (total scan time after contrast injection was $6 \mathrm{~min})$.

Gadolinium-DTPA $(0.1 \mathrm{mmol} / \mathrm{kg})$ was injected intravenously via an automatic injector at a rate of $2 \mathrm{ml} / \mathrm{s}$ followed by a saline flush. The last sequence obtained after contrast enhancement was a high-resolution axial 3D SPGR fat-saturated $\mathrm{T}_{1}$-weighted volume image (slice thickness $2.5 \mathrm{~mm}$; NEX 1, and voxel size 1.0 $\mathrm{mm}^{3}$ ). Post-processing of the dynamic images was done using the software function tool $2.6 .4 \mathrm{~b} 2$ of GE to measure the signal en- hancement ratio in order to study the kinetics of contrast enhancement within the lesions. Since accurate placement of a region of interest over the areas of most rapid and intense enhancement is critical, we used color mapping of the lesions as a guide. We also evaluated at post-processed maximum intensity projection and maximum slope of signal increase images.

\section{Image Interpretation}

Based on the American College of Radiology recommendations [6] and the diagnostic criteria identified by other investigators $[3,5,7]$, interpretation of images to classify a lesion as benign, indeterminate or malignant was done by evaluating the lesion morphology and its enhancement kinetic curves following contrast agent administration $[5,8]$. The criteria suggesting malignant lesions included a mass with indistinct, irregular, branching or spiculated borders, a mass with heterogeneous or peripheral enhancement (ring enhancement), with type II (plateau) or type III (washout) enhancement curve. Non-mass ductal enhancement with a clumped or punctuated type of morphology was characteristic of malignancy. The criteria suggestive of a benign lesion included a mass with round, oval, lobular or linear form, a mass with well-defined smooth or lobulated borders, a mass demonstrating no contrast enhancement or enhancement with a homogeneous pattern or non-enhancing internal septa, patchy parenchymal enhancement and type I (steady increase) or type II (plateau) enhancement curves. The criteria suggestive of indeterminate lesion were morphologic features and kinetic overlap between benign and malignant lesions.

The MRI findings were validated histopathologically by tissue sampling (fine needle aspiration cytology, core biopsy or lumpectomy/mastectomy). For non-sampled lesions, radiological followup by mammography, US and/or MRI was done for at least 1 year. The sensitivity, specificity, positive and negative predictive values, and the accuracy of MRI to diagnose malignant lesions with validated tissue sampling as the gold standard were then calculated using the binary classification test.

\section{Results}

Of the 58 patients, 51 underwent mammography and US examination prior to MRI and 7 had only US examination because of age $<30$ years. Of those 58 patients, MRI identified a breast mass in 40 patients, 12 had a final diagnosis of a malignant mass (5 with multifocal malignancy) and 28 a benign mass. Clinically, a mass was discovered in 7 of the 12 malignant cases and in all 28 benign cases. Surgery was performed in the 12 patients with malignancy. The histopathology of the identified cancers were well-differentiated ductal carcinoma (3); poorly infiltrating differentiated ductal carcinoma (2); well-differentiated invasive ductal carcinoma (3); well-differentiated infiltrating lobular carcinoma (3), and well-differentiated mucinous carcinoma (1). One case diagnosed as occult breast carcinoma presented with axillary lymph node metastasis and was negative clinically and radiologically (by 
Table 1. The 70 identified breast lesions by breast MRI

\begin{tabular}{|c|c|c|c|c|c|c|}
\hline & \multicolumn{2}{|c|}{$\begin{array}{l}\text { Diagnosis by tissue } \\
\text { sampling }\end{array}$} & \multicolumn{2}{|c|}{$\begin{array}{l}\text { Diagnosis by US, } \\
\text { mammography or MRI }\end{array}$} & \multirow{2}{*}{$\begin{array}{l}\text { Lesions seen } \\
\text { by MRI only } \\
\text { no final diagnosi }\end{array}$} & \multirow[t]{2}{*}{ Total } \\
\hline & malignant & benign & malignant & benign & & \\
\hline \multicolumn{7}{|l|}{ MRI diagnosis } \\
\hline Malignant & 23 & 7 & 0 & 0 & 1 & 31 \\
\hline Benign & 1 & 20 & 0 & 8 & 5 & 34 \\
\hline Indeterminate & 1 & 3 & 0 & 0 & 1 & 5 \\
\hline Total & 25 & 30 & 0 & 8 & 7 & 70 \\
\hline
\end{tabular}

US, mammography and MRI). Four of the 13 cases with a final diagnosis of breast cancer had lymph node metastases. Of the 28 patients with benign lesions, 15 had a percutaneous procedure, 5 surgery and 8 radiological followup within 6-12 months. The size of the malignant masses was $0.6-4.4 \mathrm{~cm}$ (mean of $2.5 \mathrm{~cm}$ ), whereas that of the benign masses was $0.5-6 \mathrm{~cm}$ (mean of $3.25 \mathrm{~cm}$ ).

Breast masses were not identified in 18 patients; 1 patient was diagnosed as occult breast carcinoma with metastatic axillary lymph node presentation.

The total number of lesions identified in the 40 patients was 70 . The number of lesions detected by MRI as well as mammography was 54; 17 lesions were detected by MRI, of which only 8 lesions proved malignant and 2 lesions benign after surgery (7 lesions were not proved since we had not started MRI-guided breast biopsy procedures at that time). Eight lesions diagnosed as benign by MRI were not proven by tissue sampling and remained benign by mammography and US, and were followed up for a period of 6 to 12 months.

Cancers were found in $11 \%$ of the study patients detected by MRI only. Seven other lesions (16\%) were not biopsied since MRI-guided breast biopsy had not been started at that time.

The 70 lesions detected by MRI are presented in table 1 . Of the 31 lesions diagnosed by MRI as malignant, 23 were proven malignant by tissue sampling and 7 lesions were proven benign, 4 of which were proven to be chronic granulomas. One lesion diagnosed by MRI as fibroadenoma with septations proved to be well-differentiated invasive ductal carcinoma.

The accuracy of MRI to diagnose malignant lesions proven by tissue sampling as the gold standard was $80 \%$. The sensitivity, the specificity, and the positive and negative predictive values were $96,67,71$ and $95 \%$, respectively. The sensitivity to detect recurrent malignant lesions was $100 \%$, but the specificity to differentiate a scar from a local recurrence was $50 \%$.
Table 2. The morphological features and the kinetics of the 55 lesions proven by tissue sampling as benign or malignant

\begin{tabular}{|c|c|c|}
\hline MRI criteria & $\begin{array}{l}\text { Benign } \\
\text { lesions (30) }\end{array}$ & $\begin{array}{l}\text { Malignant } \\
\text { lesions (25) }\end{array}$ \\
\hline \multicolumn{3}{|l|}{ Morphology } \\
\hline \multicolumn{3}{|l|}{ Form } \\
\hline Round & 14 & 8 \\
\hline Oval & 2 & 0 \\
\hline Lobular & 1 & 1 \\
\hline Linear & 4 & 0 \\
\hline Branching & 8 & 7 \\
\hline Spiculated & 1 & 9 \\
\hline \multicolumn{3}{|l|}{ Margin } \\
\hline Well-defined & 25 & 11 \\
\hline Indistinct & 5 & 14 \\
\hline \multicolumn{3}{|l|}{ Enhancing pattern } \\
\hline Homogeneous & 16 & 9 \\
\hline In-homogeneous & 8 & 7 \\
\hline Septated & 4 & 1 \\
\hline Ring enhancement & 0 & 8 \\
\hline \multicolumn{3}{|l|}{ Kinetics } \\
\hline \multicolumn{3}{|l|}{ Initial signal increase } \\
\hline$<50 \%$ & 4 & 0 \\
\hline $50-100 \%$ & 10 & 1 \\
\hline$>100 \%$ & 16 & 24 \\
\hline \multicolumn{3}{|l|}{ Post-initial course } \\
\hline Steady increase & 23 & 1 \\
\hline Plateau & 4 & 11 \\
\hline Washout & 3 & 13 \\
\hline
\end{tabular}

\section{Image Interpretation}

The morphological features and kinetics proven by tissue sampling as benign (30) or malignant lesions (25) are detailed in table 2 . The 4 lesions that were diagnosed as malignant but proved to be benign showed an irregular or spiculated margin and heterogeneous enhancement and were proven to be a chronic inflammation by core biopsy. One well-defined septated lesion diagnosed by 
Fig. 1. Irregular shape, ring-enhancing lesion in the right breast with contrast washout pattern (type 3 curve typical of malignancy). a Axial $\mathrm{T}_{1}$-weighted image. b Fatsaturated axial $\mathrm{T}_{1}$-weighted image after contrast enhancement. c Enhancement curve (proven well-differentiated invasive ductal carcinoma postoperatively.)

Fig. 2. Spiculated mass with heterogeneous enhancement showing strong initial signal increase with post-initial plateau (type 2 curve). a Axial $\mathrm{T}_{1}$-weighted image. b Fatsaturated axial $\mathrm{T}_{1}$-weighted image after contrast enhancement. c Enhancement curve (proven well-differentiated invasive ductal carcinoma postoperatively).

Fig. 3. Well-defined rounded mass with homogeneous enhancement showing strong initial signal increase with continued signal intensity increase (type 1 curve). a Axial $\mathrm{T}_{1}$-weighted image. b Fat-saturated axial $\mathrm{T}_{1}$-weighted image after contrast enhancement. c Enhancement curve (biopsy-proven fibroadenoma.)
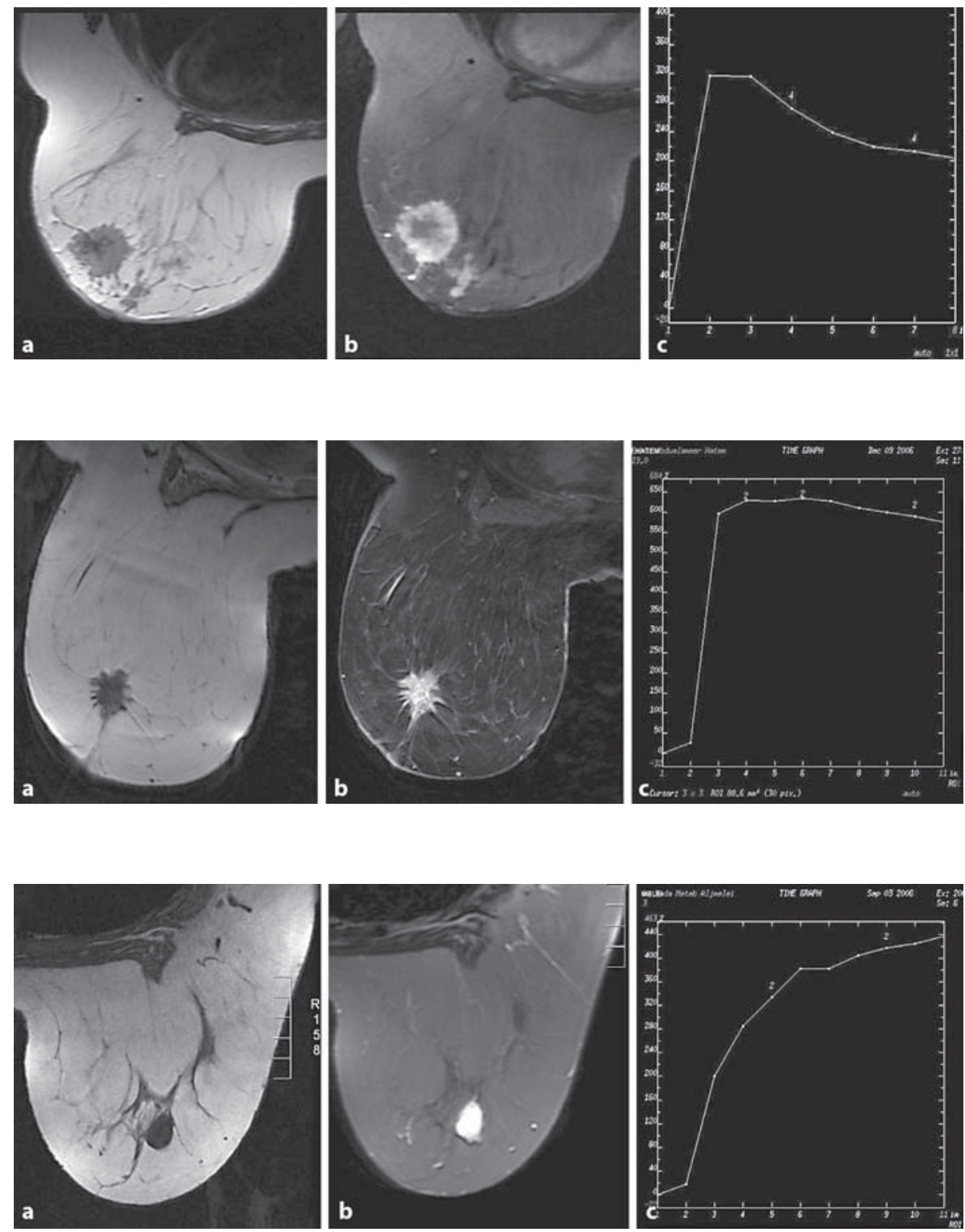

MRI as fibroadenoma proved to be invasive ductal carcinoma after surgery.

The morphemic feature of different histological groups was as follows: of the 19 ductal carcinomas, 5 (26\%) showed ring enhancement (fig. 1) and 7 (37\%) showed spiculated margins (fig. 2), and of the 5 lobular carcinomas $4(80 \%)$ showed irregular margin and 3 (60\%) showed ring enhancement. We found no significant differences among the most prevalent features of invasive cancer according to histologic subtypes. Both ductal and lobular subtypes showed irregular shape, spiculated borders and heterogeneous enhancement as their prevalent features.
The mean size of the malignant lesions was $1.6 \mathrm{~cm}$ (range, $0.6-2.7 \mathrm{~cm}$ ) for the homogeneously enhancing masses, $2.7 \mathrm{~cm}$ (range, $1.0-4.4 \mathrm{~cm}$ ) for the heterogeneously enhancing masses and $2.7 \mathrm{~cm}$ (range, $1.1-4.3 \mathrm{~cm}$ ) for the rim-enhancing masses. Carcinomas $\leq 1 \mathrm{~cm}$ were significantly more common in the homogeneously enhancing group than in the heterogeneously or rim-enhancing groups. Of the 25 carcinomas, $10(40 \%)$ were $<1 \mathrm{~cm}$. The mean size of the benign lesions was $2.2 \mathrm{~cm}$ (range, $0.5-4.0$ $\mathrm{cm}$ ) for the homogeneously enhancing benign masses (fig. 3), $3.2 \mathrm{~cm}$ (range, $0.3-6.0 \mathrm{~cm}$ ) for the heterogeneously enhancing masses and $1.8 \mathrm{~cm}$ for the septated masses (range, $1.2-2.4 \mathrm{~cm}$ ). 


\section{Discussion}

The morphology and kinetics of lesions can determine the likelihood of malignancy with very high sensitivity (ranging from 97-100\%) even in the detection of small lesions (down to $3 \mathrm{~mm}$ ) and especially in occult lesions on X-ray mammography and/or US [9]. On the other hand, specificity ranges between 50 and $80 \%$, as reported previously $[10,11]$. In our study, the accuracy of MRI was $80 \%$. Sensitivity, specificity, and positive and negative predictive values $(96,67,71$ and $95 \%$, respectively) were comparable to other studies [9-11]. Our relatively low specificity could be due to multiple factors, including limited spatial resolution of the dynamic sequences used and histologic characteristics of the lesions, possibly enhancing benign lesions as well as normal breast tissue after the administration of contrast material, which has been shown to vary in degree at different times of the menstrual cycle $[3,12]$. Equally important was the overlap of enhancement, both in terms of kinetic measurements and morphologic appearance of benign and malignant lesions, which precluded complete differentiation of lesions [3, 6-8]. Non-enhancing internal septations, a descriptor usually associated with fibroadenomas, is a sign that is no longer exclusive to benign lesions since recent results of a study by Schnall et al. [7] revealed that $47 \%$ of malignant lesions were shown to have non-enhancing internal septa. In our study, one septated mass detected proved to be well-differentiated invasive ductal carcinoma. Another problem in our study was the inclusion of inflammatory breast cancers since MRI is not really a good tool for the differentiation between benign and malignant lesions in such a clinical situation [13].

Of the 5 cases falsely reported as inflammatory carcinoma by mammography, 1 case was correctly diagnosed as chronic inflammation by MRI, whereas the other 4 cases were falsely diagnosed as invasive inflammatory carcinoma by MRI and proven by tissue sampling to be chronic inflammation possibly due to the difficulty in accurately differentiating chronic inflammation from invasive carcinoma, which was also reported by Rieber et al. [13].

Nunes et al. [14] found that none of the malignant masses had 'smooth' borders unlike our study in which $44 \%$ of the carcinomas had circumscribed borders. Nevertheless, most of these lesions had other more worrisome descriptors, such as heterogeneous or rim enhancement, which could be interpreted as malignancy as previously reported [6]. Moreover, mammographically circumscribed masses can be malignant, particularly the specific histologic subtypes of medullary, colloid and papillary carcinomas, as 5-6\% of malignant masses have been described as circumscribed [15].

The comparison of the mean pathologic size of the masses and the pattern of enhancement indicated that the mean size of carcinomas showing homogeneous enhancement was smaller $(<1 \mathrm{~cm})$ than the mean size of carcinomas showing rim or heterogeneous enhancement. The fact that $40 \%$ of the carcinomas in our study were $<1$ $\mathrm{cm}$ could be the explanation for the high prevalence of cancers with homogenous enhancement in the current series, whereas $32 \%$ of carcinomas presented with rim enhancement, which was higher than the $16 \%$ reported previously [7].

The enhancement kinetics differed significantly for benign and malignant enhancing lesions and may therefore be used as an aid in differential diagnosis (fig. 1-3). The 52\% incidence of the type 3 curve for malignant lesions and the $81.5 \%$ exhibited by the type 1 curve of our study are similar to those reported previously $[3,5,16]$. Benign lesions that showed the type 3 curve were proven to be chronic granulomas.

In our study, the main indication for MRI was to clarify equivocal lesions seen on mammography and US ( $\mathrm{n}=$ 29). MRI proved the lesions to be malignant in 3 cases and benign in 16 cases. There are reports that MRI can be used as a problem-solving tool in cases where a suspicious lesion is identified on only one mammographic view and in cases of equivocal findings of mammographic or physical examinations [8].

MRI may also help to evaluate a patient's local relapse. In our study, the $100 \%$ sensitivity to detect a lesion was similar to previously published sensitivities ranging from 93 to $100 \%$, but the $50 \%$ specificity was lower than those reported in earlier studies, ranging from 88 to $100 \%$ [17, 18]. This high false-positive rate might be attributed to the overlap of morphologic appearance of malignant lesions and scar tissue, especially the irregular margin. This could either be related to the delay between the end of treatment and breast MRI examination or to problems in MRI interpretation. Further investigations are necessary.

In patients with proven breast cancer, MRI can detect additional ipsilateral sites of breast cancer in $6-34 \%$ of cases and unsuspected contralateral cancer in $4-24 \%$ of cases [19]. In our study, MRI detected additional ipsilateral sites in $5(38 \%)$ patients and unsuspected contralateral site lesions in 2 (15\%) patients, being comparable to the above-mentioned study [19]. 
In our patients with axillary lymph node metastases who presented with negative findings in mammographic and physical examinations, primary breast cancer was detected in $75-86 \%$ by MRI compared to the $80 \%$ detection rate reported by Tilanus-Linthorst et al. [20]. Moreover, the incidence of an axillary lymph node manifestation from an occult primary breast cancer was low, with rates ranging from 0.3 to $0.8 \%$ of all patients with carcinoma of the breast at the time of presentation as reported by Solin et al. [21]. In our series, only 1 patient with a negative MRI in the initial study was diagnosed with occult breast carcinoma in the follow-up study done 5 months later after completing her 3rd cycle of chemotherapy.

\section{Conclusion}

Our overall initial experience was comparable with that of other centers. Our goal is to have specialized radiologists in breast imaging that work with a multidisciplinary treatment team. Using the ACR BI-RADS lexicon for image interpretation, improving the spatial resolution of images using a high-definition MR magnet, 8-channel breast coils, dedicated breast MR software and computeraided tomography will improve our diagnostic accuracy. Very soon MR-guided biopsy and wire localization procedures for breast masses will be introduced, which will help diagnose lesions that can be detected by MR only.

\section{References}

1 Parkin DM, Whelan SL, Ferlay J, Raymond L, Young J (eds): Cancer Incidence in Five Continents (a joint publication of IARC/ IACR, IARC Worldwide Cancer Incidence Statistics). IARC Scientific Publication No 143. Lyon, International Agency for Research on Cancer, 1997, vol VII.

-2 Skaane P, Young K, Skjennald A: Populationbased mammography screening: comparison of screen-film and full-field digital mammography with soft-copy reading Oslo I study. Radiology 2003;229:877-884.

-3 Kuhl CK: The current status of breast MR imaging. Part I. Choice of technique, image interpretation, diagnostic accuracy, and transfer to clinical practice. Radiology 2007; 244:356-378.

4 Morris EA: Diagnostic breast MR imaging: current status and future directions. Radiol Clin North Am 2007;45:863-880, vii.

5 Macura KJ, Ouwerkerk R, Jacobs MA, Bluemke DA: Patterns of enhancement on breast MR images: interpretation and imaging pitfalls. RadioGraphics 2006;26:17191734.

6 ACR practice guideline for the performance of magnetic resonance imaging (MRI) of the breast. http://www.greenemedicalimaging. com/services/BreastMRI.htm.

7 Schnall MD, Blume J, Bluemke DA, DeAngelis GA, DeBruhl N, Harms S, Heywang-Köbrunner SH, Hylton N, Kuhl CK, Pisano ED, Causer P, Schnitt SJ, Thickman D, Stelling CB, Weatherall PT, Lehman C, Gatsonis CA: Diagnostic architectural and dynamic features at breast MR imaging: multicenter study. Radiology 2006;238:42-45.
8 Kuhl CK, Mielcareck P, Klaschik S, Leutner C, Wardelmann E, Gieseke J, Schild HH: Dynamic breast MR imaging: are signal intensity time course data useful for differential diagnosis of enhancing lesions? Radiology 1999;211:101-110.

-9 Fischer U, Vosshenrich R, Probst A, Burchhardt H, Grabbe E: Breast carcinoma: effect of preoperative contrast-enhanced MR imaging on the therapeutic approach. Radiology 1999;213:881-888.

10 Lee CH: Problem solving MR imaging of the breast. Radiol Clin North Am 2004;42:919934.

11 MARBIS Study Group: Screening with magnetic resonance imaging and mammography of a UK population at high familial risk of breast cancer: a prospective multicentre cohort study (MARBIS). Lancet 2005;365: 1769-1778.

12 Muller-Schimpfle M, Ohmenhauser K, Stoll P, Dietz K, Claussen CD: Menstrual cycle and age: influence of parenchymal contrast medium enhancement in MR imaging of the breast. Radiology 1997;203:145-149.

13 Rieber A, Tomczak RJ, Mergo PJ, Wenzel V, Zeitler H, Brambs HJ: MRI of the breast in the differential diagnosis of mastitis versus inflammatory carcinoma and follow-up. J Comput Assist Tomogr 1997;21:128-132.
14 Nunes LW, Schnall MD, Orel SG, Hochman MG, Langlotz CP, Reynolds CA, Torosian $\mathrm{MH}$ : Breast MR imaging: interpretation model. Radiology 1997;202:833-841.

15 Kopans DB: Breast Imaging, ed 2. Philadelphia, Lippincott-Raven, 1998.

$\checkmark 16$ Kuhl CK, Schild HH, Morakkabati N: Dynamic bilateral contrast-enhanced MR imaging of the breast: trade-off between spatial and temporal resolution. Radiology 2005; 236:789-800.

17 Orel SG: High-resolution MR imaging for the detection, diagnosis, and staging of breast cancer. RadioGraphics 1998;18:903912.

18 Rieber A, Merkle E, Zeitler H, Rieber, Görich J, Kreienberg R, Brambs HJ, Tomczak R: Value of MR mammography in the detection and exclusion of recurrent breast carcinoma. J Comput Assist Tomogr 1997;21:780-784.

19 Liberman L, Morris EA, Kim CM, Kaplan JB, Abramson A, Menell JH, Van Zee KJ, Dershaw DD: MR imaging findings in the contralateral breast of women with recently diagnosed breast cancer. AJR 2003;180: 333-341.

20 Tilanus-Linthorst MM, Obdeijn AI, Bontenbal M, Oudkerk M: MRI in patients with axillary metastases of occult breast carcinoma. Breast Cancer Res Treat 1997;44:179-182.

21 Solin LJ, Fowble B, Goodman RL, Glick JH, Rosato EF: Special Considerations in Breast Cancer Treatment: A Comprehensive Guide to Management. St. Louis, Mosby Year Book, 1991, pp 523-528. 